\title{
Antiplatelet Agents for Stroke Prevention
}

\author{
Samuel Yip • Oscar Benavente
}

Published online: 15 July 2011

(C) The American Society for Experimental NeuroTherapeutics, Inc. 2011

\begin{abstract}
Stroke is one of the leading causes of disability and death. Ischemic stroke is a syndrome with heterogeneous mechanisms and multiple etiologies, rather than a singularly defined disease. Approximately one third of ischemic strokes are preceded by another cerebrovascular ischemic event. Stroke survivors are at high risk of vascular events (i.e., cerebrovascular and cardiovascular events), particularly during the first several months after the ischemic event. The use of antiplatelet agents remains the fundamental component of secondary stroke prevention. Based on the available data, antiplatelet agents should be used for patients with noncardioembolic stroke. The use of combination therapy (aspirin plus clopidogrel) has not been proven to be effective or safe to use for prevention of early stroke recurrence or in long-term treatment. There is no convincing evidence that any of the available antiplatelet agents are superior for a given stroke subtype. Currently, the uses of aspirin, clopidogrel, or aspirin combined with extended release dipyridamole are all valid alternatives after an ischemic stroke or transient ischemic attack. However, to maximize the effects of these agents, the treatment should be initiated as early as possible and be continued on a lifelong basis.
\end{abstract}

Electronic supplementary material The online version of this article (doi:10.1007/s13311-011-0060-2) contains supplementary material, which is available to authorized users.

S. Yip $(\bowtie) \cdot$ O. Benavente Division of Neurology, Brain Research Centre, University of British Columbia, Stroke Program, Vancouver, British Columbia V5Z 1M9, Canada e-mail: samyip100@gmail.com
Keywords Stoke $\cdot$ Prevention $\cdot$ Antiplatelets $\cdot$ Clinical trials . Aspirin $\cdot$ Clopidogrel $\cdot$ Dipyridamole

\section{Introduction}

Antiplatelet Agents in Secondary Stroke Prevention

Each year, approximately 795,000 people in the United States suffered a stroke of which 185,000 are recurrent attacks [1]. Approximately $20 \%$ of patients who have had a transient ischemic attack (TIA) or minor stroke will have a recurrent vascular event within 1 year [2]. The risk of recurrent ischemic stroke is especially high within the first week after a minor stroke or TIA [3]. Numerous efforts have been placed to identify treatments that may decrease the risk of stroke recurrences. Aspirin has been shown to be effective in secondary stroke prevention in the 1970s and forms the cornerstone of our current ischemic stroke prevention strategy. With the development of newer antiplatelet agents, such as the thienopyridines (i.e., ticlopidine and clopidogrel) and dipyridamole, our armamentarium for secondary stroke prevention has significantly increased. In recent years, tremendous efforts have been placed in conducting randomized clinical trials to better understand the efficacy and safety of each antiplatelet agent and the comparison between each, so that a systematic approach of selection of antiplatelet agents could be established. These trials form our current evidence-based approach of antiplatelet therapies for secondary stroke prevention $[4,5]$. In this article, we will review and discuss the following topics: 1) the mechanisms of action of different antiplatelet agents currently approved for stroke prevention, 2) the clinical trials that 
have led to the development of the evidence-based practice in antiplatelet agents for secondary stroke prevention, 3) the efficacy of antiplatelet agents for secondary stroke prevention in different stroke subtypes (i.e., large artery atherosclerosis and small vessel lacunar infarct), 4) the significance of antiplatelet resistance, and 5) future directions on clinical trial design for antiplatelet agents in secondary stroke prevention.

Mechanism of Action of Current Antiplatelets Used Clinically

Antiplatelet agents that are currently approved by the Food and Drug Administration for secondary stroke prevention include aspirin, clopidogrel, and dipyridamole. These agents act at different sites of platelet aggregation pathway to inhibit platelet activation.

Aspirin is an irreversible cyclooxygenase (COX) inhibitor, which selectively acetylates the hydroxyl group of the COX enzyme resulting in inhibition of conversion of arachidonate to prostaglandin $\mathrm{G} 2 / \mathrm{H} 2$, and thromboxane A2. This leads to irreversible inhibition of platelet aggregation [6].

Clopidogrel is a thienopyridine derivative that inhibits the binding of adenosine 5-diphosphate to glycoprotein IIb/IIIa preventing the binding of fibrinogen to this receptor [7].

Dipyridamole is postulated to have multiple mechanisms of action, and primarily inhibits platelet phosphodiesterase resulting in an increase in intraplatelet cyclic adenosine monophosphate level, thereby potentiating the plateletinhibitory actions of prostacyclin. Dipyridamole also acts by directly releasing eicosanoid from vascular endothelium, and it inhibits cellular uptake and metabolism of adenosine, which again inhibits platelet aggregation [8].

Due to their different mechanisms of action, it is postulated that these antiplatelet agents may provide different degrees of vascular protection. In addition, combination therapy as compared to monotherapy may be more effective in reducing vascular events. This has led to multiple clinical trials studying the efficacy of these agents in ischemic stroke prevention.

\section{Clinical Trials for Prevention of Early Stroke Recurrences}

Recent epidemiological data suggest that patients who have suffered from an ischemic stroke are at high risk of recurrent ischemic events in the immediate poststroke period [3]. Recurrent risk is associated with the underlying stroke etiology [9-11]. The estimated risk of stroke recurrence is 10 to $20 \%$ within the first 3 months. Aspirin and combination of aspirin plus clopidogrel have been studied in randomized, placebo-controlled trials for prevention of early stroke recurrence.
Aspirin

The Chinese Acute Stroke Trial (CAST) [12] and International Stroke Trial (IST) [13] randomized a total of 40,090 patients into receiving aspirin (160-300 mg) vs placebo within the first $48 \mathrm{~h}$ of stroke symptom onset. In a prespecified meta-analysis of the Chinese Acute Stroke Trial and the International Stroke Trial data, early aspirin produced a reduction of 7 per 1000 of fatal or nonfatal recurrent ischemic stroke (320 [1.6\%] aspirin vs 457 [2.3\%] control; $2 p<0.000001$ ) [14]. There is a small increase of 2 per 1000 of hemorrhagic stroke (202 [1.0\%] aspirin vs 167 $[0.8 \%]$ control, $2 p=0.07)$. Aspirin also produced a reduction of 9 per 1000 of further stroke or death (427 [8.2\%] aspirin vs 516 [9.1\%] control, $2 \mathrm{P}<0.001)$. Based on these data, the current recommendation of immediate use of aspirin for prevention of early stroke recurrence was established [5].

\section{Aspirin and Clopidogrel}

The Fast Assessment of Stroke and Transient Ischemic Attack to Prevent Early Recurrence (FASTER) trial was designed to assess (in a $2 \times 2$ factorial design) whether: 1 ) combination of aspirin plus clopidogrel $v s$ aspirin monotherapy or 2) simvastatin $v s$ placebo, if started within $24 \mathrm{~h}$ of symptom onset would reduce the risk of recurrent stoke within 90 days after a TIA or minor stroke [15]. The trial planned to enroll 500 patients, but failed to recruit greater than its prespecified enrollment success rate for 12 months. This led to the steering committee's decision to close the trial prior to completion. In the final analysis of 392 patients, the Fast Assessment of Stroke and Transient Ischemic Attack to Prevent Early Recurrence trial showed a trend toward benefit with the combination of aspirin plus clopidogrel for prevention of early stroke recurrence. 14 (7.1\%) patients on aspirin plus clopidogrel had a stroke within 90 days compared with $21(10.8 \%)$ patients on placebo (risk ratio [RR], 0.7 [95\% confidence interval (CI), 0.3-1.2]; $p=0.19$ ). Two patients on clopidogrel had intracranial hemorrhage compared with none on placebo (absolute risk increase (ARI), $1 \%$ [95\% CI, $-0.4-2.4] ; p=0.5$ ).

Two ongoing trials, the Platelet-Oriented Inhibition in New TIA and Minor Ischemic Stroke (POINT) trial, and the Clopidogrel in High-risk Patients with Acute Nondisabling Cerebrovascular Events (CHANCE) study have been set out to determine whether early use of combination of aspirin and clopidogrel is effective in preventing major ischemic vascular events (ischemic stroke, myocardial infarction, and ischemic vascular death) at 90 days. The Platelet-Oriented Inhibition in New TIA and Minor Ischemic Stroke study will randomize 5000 patients in North America and is planned to finish recruitment in 2016. The 
Clopidogrel in High-risk Patients with Acute Non-disabling Cerebrovascular Events study is a similar study being carried out in China. It is expected to be completed by the second quarter of 2011 [16].

Clinical Trials for Prevention of Late Stroke Recurrences

Aspirin vs Placebo

There have been 11 clinical trials comparing the effect of aspirin to placebo for secondary stroke prevention. Ten of the 11 studies have been reviewed thoroughly by Algra and van Gijn [17] in 1996. Most of these studies included patients with stroke or TIA. Doses of aspirin used in these studies ranged from 30 to $1300 \mathrm{mg}$ per day. The time from index event to enrollment varied from 1 week to 1 year. Some studies excluded patients with cardioembolic source and high-grade carotid stenosis requiring carotid endarterectomy. Only 1 study reported inclusion of small vessel lacunar infarct. Otherwise, no specific data on the subtype of stroke is reported in most studies.

Algra and van Gijn [17] performed a meta-analysis of the 10 clinical trials that included 6171 patients. The primary endpoint was defined as the composite outcome of vascular death, stoke, or myocardial infarction. The meta-analysis revealed a $13 \%$ relative risk reduction of aspirin recipients compared to a placebo. Moreover, it was concluded that the beneficial effect of aspirin was seen in doses $>30 \mathrm{mg}$ /day.

The 11th study was the European stroke prevention study 2 (ESPS-2) study, which was a double-blind, randomized placebo-controlled trial testing the effect of combination of dipyridamole and aspirin [18]. This study was published in 1996 and had a $2 \times 2$ factorial design, included a placebo arm and an aspirin alone arm. In the aspirin (50 mg/day) and placebo arms, a total of 3300 patients were included. Primary endpoint was stroke, death, or both stroke and death. Aspirin significantly reduces stroke, and stroke or death when compared to a placebo control (Relative Risk Reduction [RRR], 18.1\% standard error [SE], 7.2; $p=0.013$ and RRR, $13.2 \%$ SE $5.8 ; p=0.016$ ). This data is very similar to that reported in Algra and van Gijn's [17] meta-analysis, and it supports the modest effects of aspirin for secondary stroke prevention. These data from the cornerstone of aspirin use in modern ischemic secondary stroke prevention strategy. Based on these data, a further placebo study is considered unethical, and therefore aspirin has become the gold standard for therapy in subsequent randomized control trials.

The Antithrombotic Trialists' Collaboration (ATTC) has also confirmed the previously described observations. In their most recent meta-analysis, individual participant data comparing long-term aspirin $v s$ a control from 16 secondary prevention trials with 17,000 individuals at an average high risk was published. Composite outcome of myocardial infarction, stroke, or vascular death was defined as the primary endpoint. There were 3306 patients who reached the primary endpoint. Aspirin provided an ARR of serious vascular events of $1.5 \%$ per year (RR, 0.81 [95\% CI, 0.750.87]; $p<0.00001)$. Hemorrhagic stroke was increased with aspirin use (RR, 1.67 [95\% CI, 0.81-3.44]; $p=0.01$ ). There was a significant reduction of total ischemic stroke (RR, 0.78 [95\% CI, 0.61-0.99]; $p=0.04$ ) [19].

\section{Antiplatelet Agents in Nonvalvular Atrial Fibrillation}

Oral anticoagulation is the treatment of choice for patients with atrial fibrillation at high risk of stroke [20]. Warfarin reduces stroke by two-thirds compared with placebo treatment and reduces stroke by $45 \%$ when compared with aspirin in patients with nonvalvular atrial fibrillation. However, anticoagulation also increases the risk of major bleeding by approximately $70 \%$ compared with aspirin. The response to oral anticoagulation therapy is variable and requires regular monitoring. These factors have led to underutilization of warfarin for stroke prevention in atrial fibrillation. As a result, combination aspirin and clopidogrel was tested for stroke prevention in atrial fibrillation as an alternative treatment in the Atrial fibrillation Clopidogrel Trial with Irbesartan for prevention of Vascular Event (ACTIVE-W) and the ACTIVE-A trials [21, 22].

ACTIVE-W was a randomized, open label clinical trial allocating patients with a history of atrial fibrillation plus 1 or more risk factors for stroke to receive either oral anticoagulation therapy (target international normalized ratio [INR], 2.0-3.0; $\mathrm{n}=3371)$ or clopidogrel $(75 \mathrm{mg} /$ day $)$ plus aspirin $(75-100 \mathrm{mg} /$ day; $\mathrm{n}=3335)$. Outcome events were adjudicated by a blinded committee. Primary outcome was the first occurrence of stroke, non-central nervous system systemic embolus, myocardial infarction, or vascular death. After a median follow-up of 1.28 years, the study was terminated early because of superiority of oral anticoagulation therapy over the dual antiplatelet regimen (annual risk of primary outcome, $3.93 \%$ vs $5.60 \%$, respectively; RR, 1.44 [95\% CI, $1.18-1.76] ; p=0.0003)$. Annual rate of major hemorrhage was $2.42 \%$ in the aspirin plus clopidogrel group and $2.21 \%$ in the oral anticoagulation group (RR, 1.10 [95\% CI, $0.83-$ $1.45] ; p=0.53)$. There was a small, but statistically significant, increased annual risk of hemorrhagic stroke with oral anticoagulation $v s$ aspirin plus clopidogrel $(0.36 \%$ vs $0.12 \%$; $p=0.036$ ). This trial confirms the previous observation of superiority of oral anticoagulation for stroke prevention in patients with atrial fibrillation and supports current recommendation. For patients who are already on anticoagulation and have had no significant side effects, oral anticoagulation is the treatment of choice. They should not be switched to a combination therapy of aspirin and clopidogrel. For those who are warfarin naive, warfarin is the standard of therapy. 
In the ACTIVE-A study, 7554 patients with atrial fibrillation, in whom anticoagulation was considered unsuitable, were randomized to receive either clopidogrel (75 $\mathrm{mg} ; \mathrm{n}=3772)$ or placebo $(\mathrm{n}=3782)$ in addition to aspirin (75 to $100 \mathrm{mg}$ ) daily (ACTIVE Investigators, 2009). The primary endpoint was stroke, myocardial infarction, systemic embolism, and vascular death. During a median follow-up of 3.6 years, clopidogrel plus aspirin combination resulted in a statistically significant reduction of primary outcome when compared to aspirin alone $(6.8 \%$ and $7.6 \%$ per year, respectively; RR, 0.89 [95\% CI, 0.81 to 0.98]; $p=0.01$ ). This benefit was mostly due to a reduction in ischemic stroke with clopidogrel plus aspirin (absolute risk reduction [ARR], $0.9 \%$ per year; RR, 0.68 [95\% CI, 0.57-0.80]). Major bleeding occurred at a rate of $2 \%$ per year in clopidogrel plus aspirin and $1.3 \%$ per year in the aspirin alone group (RR, 1.57 [95\% CI, 1.29-1.92]; $p<0.001$ ). There was a significant increase in risk of intracranial hemorrhage with clopidogrel plus aspirin $v$ s aspirin alone $(0.4 \%$ and $0.2 \%$ per year, respectively; RR, 1.87 [95\% CI, 1.19-2.94]; $p=$ $0.006)$. With the combination of major vascular events (the primary outcome) and major hemorrhage, there was no significant difference between the overall event rate with aspirin plus clopidogrel and aspirin alone (RR, 0.97 [95\% CI, 0.89-1.06]; $p=0.54$ ) (Tables 1, 2 and 3).

\section{Antiplatelet Agents in Noncardioembolic Stroke}

Since the publication of North American Symptomatic Carotid Endarterectomy Trial (NASCET) and the Medical Research Council (MRC) European Carotid Surgery Trial
(ECST), carotid endarterectomy has become the standard of therapy for symptomatic high-grade carotid stenosis [23, 24]. With the meta-analysis of the Atrial Fibrillation Trialist, anticoagulation has become the routine therapy for secondary stroke prevention in patients with nonvalvular atrial fibrillation [20]. Therefore, most of the recent trials examining the efficacy of antiplatelet agents on secondary prevention of stroke have excluded patients with cardioembolic causes and high-grade symptomatic carotid stenosis. As shown in Table 4, the majority of patients enrolled in recent clinical trials have small vessel disease, large artery atherosclerosis (excluding those with high-grade symptomatic carotid stenosis suitable for surgery), and cryptogenic stroke. These varied stroke subtypes have been grouped together and are classified as noncardioembolic or presumed arterial origin strokes. This group represents the study population in most of our recent clinical trials.

\section{Aspirin vs Anticoagulation}

Prior to the Warfarin Aspirin Recurrent Stroke Studies (WARSS), the role of antiplatelet $v s$ anticoagulant was unclear in patients with noncardioembolic stroke. The Stroke Prevention in Reversible Ischemia Trial (SPIRIT) compared efficacy of warfarin (target INR, 3-4.5) and aspirin $(30 \mathrm{mg} /$ day) in stroke prevention in patients with a history of TIA or minor ischemic stroke of presumed arterial origin. The trial was prematurely stopped due to excess hemorrhage rate in the warfarin arm (hemorrhage rate $[\mathrm{HR}], 9.3$ [95\% CI, 4.0-22]). Moreover, ischemic events rate were similar in both treatment arms ([HR], 1.03 [95\% CI, 0.6-1.75]) [25].

Table 1 Antiplatelet in Prevention of Early Recurrent Stroke

\begin{tabular}{|c|c|c|c|c|c|c|c|c|}
\hline Study & Population & ETR & $\begin{array}{l}\text { Stroke } \\
\text { Subtype }\end{array}$ & $\begin{array}{l}\text { Follow- } \\
\text { up }\end{array}$ & Treatment & Primary Outcome & Efficacy & Safety \\
\hline CAST, 1997 & $\begin{array}{l}\text { 21106, suspected } \\
\text { AIS }\end{array}$ & Within $48 \mathrm{~h}$ & $\mathrm{Lac}=30 \%{ }^{\mathrm{a}}$ & 4 weeks & $\begin{array}{c}\text { ASA } 160 \mathrm{mg} \\
\text { vs placebo }\end{array}$ & $\begin{array}{l}\text { 1) Death from } \\
\text { any cause } \\
\text { 2) Death or } \\
\text { dependence } \\
\text { at discharge }\end{array}$ & $\begin{array}{l}\text { 1) } \mathrm{ARR}, 0.6 \% \text {, } \\
(2 p=0.04) \text {; } \\
\text { 2) ARR, } 1.1 \% \\
(2 p=0.08)\end{array}$ & $\mathrm{HS}=0.2 \%$ \\
\hline IST & $\begin{array}{l}\text { 19432, suspected } \\
\text { AIS }\end{array}$ & Within $48 \mathrm{~h}$ & $\mathrm{Lac}=24 \%{ }^{\mathrm{a}}$ & 6 months & $\begin{array}{c}\text { ASA } 300 \mathrm{mg} \\
\text { vs placebo }\end{array}$ & $\begin{array}{l}\text { 1) Death within } \\
14 \text { days } \\
\text { 2) Death or } \\
\text { dependency } \\
\text { at } 6 \text { months }\end{array}$ & $\begin{array}{l}\text { 1) } \mathrm{ARR}, 0.4 \% \\
\text { 2) } \mathrm{ARR}, 1.3 \% \\
(2 p=0.07)\end{array}$ & $\mathrm{HS}=0.1 \%$ \\
\hline FASTER & $\begin{array}{r}\text { 392, TIA or } \\
\text { minor AIS }\end{array}$ & Within $24 \mathrm{~h}$ & $\begin{array}{l}\mathrm{Lac}=28.8 \%^{\mathrm{b}} \\
\mathrm{CE}=6.6 \% \\
\mathrm{LAA}=24.0 \% \\
\mathrm{Cry}=36.7 \%\end{array}$ & 3 months & $\begin{array}{l}\text { Placebo } \\
\quad v s \text { clopidogrel } \\
\quad(75 \mathrm{mg})\end{array}$ & $\begin{array}{l}\text { Total stroke } \\
\text { within } 90 \text { days }\end{array}$ & $\begin{array}{c}\text { ARR, 3.8\%; } \\
\quad(p=0.19)\end{array}$ & $\mathrm{HS}=1 \%$ \\
\hline
\end{tabular}

$\mathrm{AIS}=$ acute ischemic stroke; $\mathrm{ARR}=$ absolute risk reduction; $\mathrm{ASA}=$ aspirin; $\mathrm{CAST}=$ Chinese Acute Stroke Trial; $\mathrm{CE}=\mathrm{Cardioembolism}$; Cry $=$ cryptogenic stroke; ETR = event to randomization; FASTER = Fast Assessment of Stroke and Transient Ischemic Attack to Prevent Early Recurrence trial; HS = hemorrhagic stroke; IST $=$ International Stroke Trial; LAA = large artery atherosclerosis; LAC $=$ lacunar stroke; $2 p=2$ tail test

${ }^{\mathrm{a}}$ Based on Oxfordshire Community Stroke Project (OCSP) classification; ${ }^{\mathrm{b}}$ Based on Trial of Org 10172 in Acute Stroke Treatment (TOAST) criteria 
Table 2 ASA vs Anticoagulation for Secondary Stroke Prevention in Noncardioembolic Stroke

\begin{tabular}{|c|c|c|c|c|c|c|c|c|c|c|c|}
\hline \multirow[t]{2}{*}{ Study } & \multirow[t]{2}{*}{$\begin{array}{l}\text { Patient } \\
\text { Population }\end{array}$} & \multirow[t]{2}{*}{ ETR } & \multirow[t]{2}{*}{$\begin{array}{l}\text { Stroke } \\
\text { Subtype (\%) }\end{array}$} & \multirow[t]{2}{*}{ Follow-up } & \multicolumn{2}{|l|}{ Treatment } & \multirow[t]{2}{*}{ Primary Outcome } & \multicolumn{2}{|l|}{ Efficacy } & \multicolumn{2}{|c|}{$\begin{array}{l}\text { Major } \\
\text { Hemorrhage }\end{array}$} \\
\hline & & & & & ASA (mg/day) & AC INR & & ASA & $\mathrm{AC}$ & ASA & $\mathrm{AC}$ \\
\hline SPIRIT & $\begin{array}{l}1316 \text { with } \\
\text { IS or TIA }\end{array}$ & $\begin{array}{l}\text { Within } \\
6 \text { months }\end{array}$ & NS & 14 months & 30 & $3-4.5$ & $\begin{array}{l}\text { Vascular death, } \\
\text { nonfatal stroke, } \\
\text { nonfatal MI, or } \\
\text { nonfatal major } \\
\text { hemorrhage }\end{array}$ & $\begin{array}{l}5.4 \% \\
\mathrm{HR}, 2.3 ; 95 \% \mathrm{CI}, \mathrm{H} \\
\quad 1.6-3.5\end{array}$ & $\begin{array}{l}12.4 \% \\
\text { HR,9.3; 95\% CI, } \\
\quad 4.0-22\end{array}$ & $0.9 \%$ & $8.1 \%$ \\
\hline WARSS & 2206 with IS & $\begin{array}{l}\text { Within } \\
30 \text { days }\end{array}$ & $\begin{array}{l}\mathrm{LAC}=55.5 \\
\mathrm{LAA}=13.1 \\
\mathrm{Cry}=25.5^{\mathrm{a}}\end{array}$ & 2 years & 325 & $1.4-2.8$ & $\begin{array}{l}\text { Recurrent IS } \\
\text { or death }\end{array}$ & $\begin{array}{l}16.0 \% \\
\text { HR, } 1.13 ; 95 \% \text { CI, } \\
\quad 0.92-1.38 ; \\
p=0.25\end{array}$ & $17.8 \%$ & $\begin{array}{l}2.2^{*} \\
\mathrm{RR}, 1 . \\
0.93\end{array}$ & $\begin{array}{l}1.5^{\mathrm{c}} \\
95 \% \mathrm{CI},\end{array}$ \\
\hline ESPRIT & $\begin{array}{l}1068 \text { with IS } \\
\text { or TIA }\end{array}$ & $\begin{array}{l}\text { Within } \\
6 \text { months }\end{array}$ & $\begin{array}{l}\mathrm{LVD}=33 \\
\mathrm{SVD}=48^{\mathrm{b}}\end{array}$ & $\begin{array}{l}\text { Mean, } \\
4.6 \text { years }\end{array}$ & $30-325$ & $2-3$ & $\begin{array}{l}\text { Vascular death, } \\
\text { nonfatal stroke, } \\
\text { nonfatal MI, or } \\
\text { nonfatal major } \\
\text { hemorrhage }\end{array}$ & $\begin{array}{l}18 \% \\
\text { HR, } 1.02 ; 95 \% \mathrm{CI} \\
0.77-1.35 ; p=0.25\end{array}$ & $\begin{array}{l}19 \% \\
5\end{array}$ & $\begin{array}{r}3.4 \% \\
\mathrm{HR}, 2 . \\
1.48\end{array}$ & $\begin{array}{l}8.4 \% \\
95 \% \mathrm{CI}, \\
43\end{array}$ \\
\hline
\end{tabular}

$\mathrm{AC}=$ anticoagulation; $\mathrm{ASA}=$ aspirin; $\mathrm{CI}=$ confidence interval; Cry $=$ cryptogenic stroke; ESPRIT $=$ European/Australasian Stroke Prevention in Reversible Ischaemia Trial; ETR = Event to Randomization; HR = hazard ratio; INR = international normalized ratio; IS = ischemic stroke; LAA = large artery atherosclerosis; LAC = lacunar; $\mathrm{LVD}=$ Large Vessel Disease; $\mathrm{MI}=$ myocardial infarction; $\mathrm{NS}=$ not specified; RR = rate ratio; $\mathrm{SPIRIT}=$ Stroke Prevention in Reversible Ischemia Trial; SVD = Small Vessel Disease; TIA = transient ischemic attack; TTE = time to enrollment; WARSS = Warfarin Aspirin Recurrent Stroke Studies

${ }^{a}$ Based on TOAST Criteria; ${ }^{b}$ classification based defined by the study; ${ }^{c}$ unit $=$ per 100 patients-year

WARSS was a randomized double-blind, multicenter trial comparing the efficacy of warfarin (INR, 1.4 to 2.8 ) to aspirin $(325 \mathrm{mg} /$ day $)$ in stroke prevention in patient with noncardioembolic stroke [26]. A total of 2206 patients with an ischemic stroke within the previous 30 days were enrolled. For a 2-year follow-up period, no statistical difference was found between the treatment groups in the primary endpoint of death or recurrent stroke (warfarin $[17.8 \%]$ vs aspirin [16\%]; $p=0.25)$. The major hemorrhage rates were 2.22 per 100 patient-years in the warfarin group vs 1.49 per 100 patient-years in the aspirin group (OR, 1.48 [95\% CI, 0.93-2.44]; $p=0.10$ ). Based on these data, it was concluded that oral anticoagulation with target INR of 2 to 3 is not more effective than aspirin for secondary prevention after noncardioembolic ischemic stroke.

Additional data from the European/Australasian Stroke Prevention in Reversible Ischaemia Trial (ESPRIT) supports the conclusion previously described [27]. The ESPRIT is an open label, non-blinded study examining the effects of medium intensity of oral anticoagulants (INR, 2-3) vs aspirin (30 to $325 \mathrm{mg} /$ day) in secondary stroke prevention of presumed arterial origin. There were 1068 patients who were randomized. The primary endpoint was defined as the composite of death from all vascular causes, nonfatal stroke, nonfatal myocardial infarction, or major bleeding complication, whichever occurred first. There was no significant difference observed in the number of primary endpoint events between the two treatment groups (hazard ratio [HR], 1.02 [95\% CI, 0.77-1.35]); however, the risk of major bleeding was significantly higher in the warfarin group (HR, 2.56 [95\% CI, 1.48-4.43]).

\section{Aspirin Plus Dipyridamole}

There are 6 clinical trials comparing the effects of aspirin and dipyridamole $v s$ aspirin in secondary stroke prevention, and 4 of the 6 studies compared aspirin with aspirin plus immediate-release dipyridamole. A meta-analysis was performed by Verro et al. [28] using data from these 4 studies [28]. A total of 1611 patients were included in the meta-analysis. It showed no significant difference in either the stroke-alone endpoint (RR, 0.83 ; 95\% CI, 0.59-1.15) or the composite outcome (RR, 0.95 [95\% CI, 0.75-1.19]) of stroke, death, or myocardial infarction (MI).

Two large randomized trials (the ESPS2 and the ESPRIT), have examined the effect of aspirin and extended-release dipyridamole (ERDP) on secondary stroke prevention. The ESPS2 trial, as stated earlier, was a randomized, double-blind, placebo-controlled trial with a $2 \times 2$ factorial design [18]. Patients having events in the preceding 3 months were randomized between treatment with aspirin alone (25 $\mathrm{mg}$ twice a day [bid]; $\mathrm{n}=1649)$, ERDP alone (200 mg bid; $\mathrm{n}=1654$ ), the 2 agents in a combine formulation $(n=1650)$, or placebo $(n=1649)$. Primary endpoints were stroke, death, or stroke and death together. For a 2-year follow-up period, all 3 treatment arms significantly reduced the risk of recurrent stroke when compared to placebo (aspirin: RRR, 18.1; SE, 7.2; $p=0.013$; ERDP: RRR, 16.3; SE, 7.2; $p=0.039$; aspirin-ERDP: RRR, 37 ; SE, $6.0 ; p<0.001)$. None of the treatments significantly reduced the risk of death alone or the risk of fatal stroke. Comparisons of the aspirin-ERDP $v s$ aspirin alone yielded a statistically significant reduction of stroke risk of $23.1 \%$ 


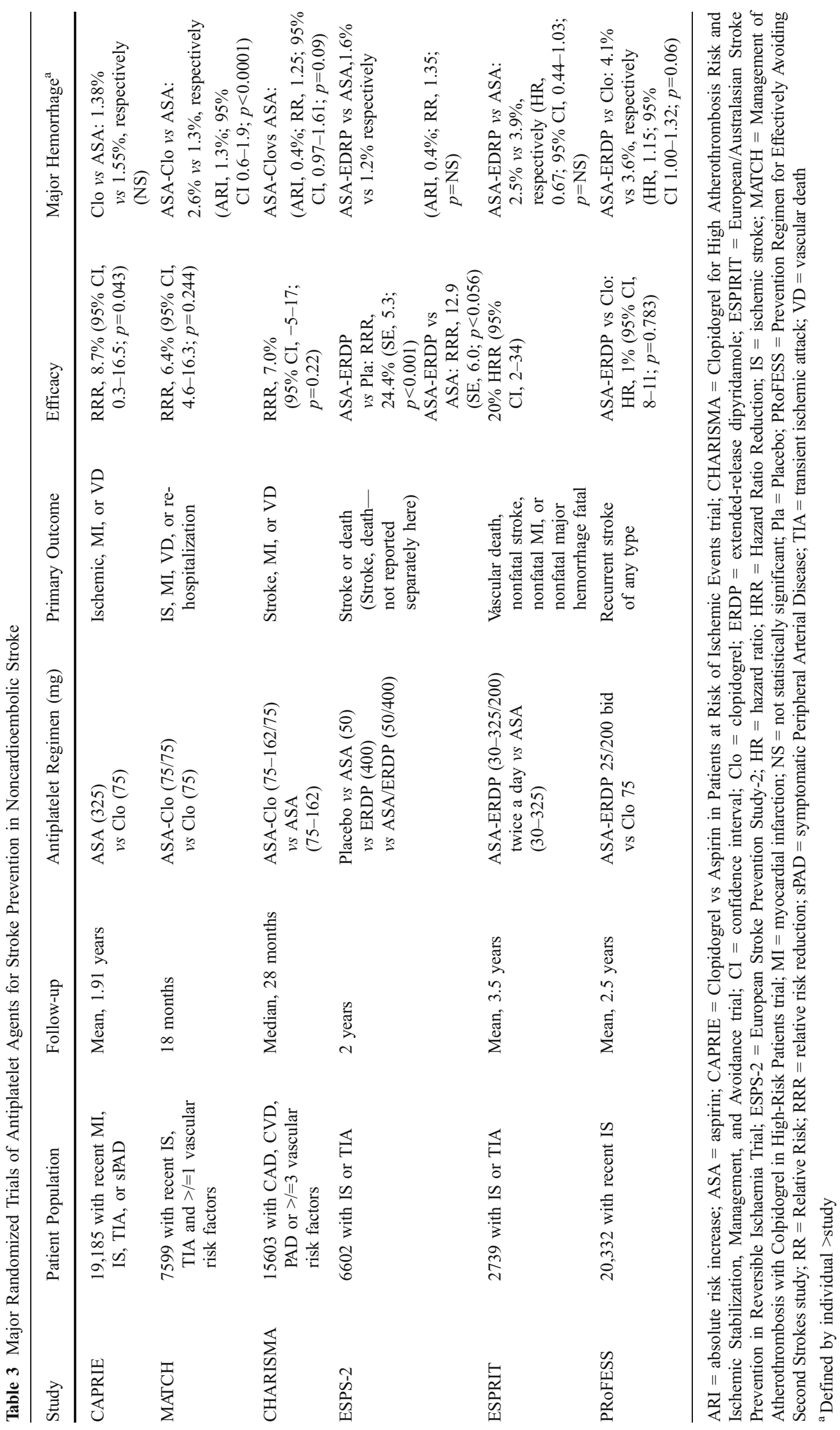


Table 4 Comparison of Trial Population Characteristics between the Major Randomized Trials

\begin{tabular}{|c|c|c|c|c|c|}
\hline & ESPS-2 & $\begin{array}{l}\text { CAPRIE } \\
\text { (Stroke Subgroup) }\end{array}$ & ESPRIT & MATCH & PRoFESS \\
\hline Enrollment Period & 1989-1993 & 1992-1995 & $1997-2005$ & $2000-2002$ & 2003-2008 \\
\hline $\mathrm{N}$ & 6605 & 6431 & 2739 & 7599 & 20,332 \\
\hline Mean age & 67 & 65 & 63 & 66 & 66 \\
\hline ETR & $<3$ months & $\geq 1$ week to $\geq 6$ months & $<6$ months & $<3$ months & $<90$ days \\
\hline$<1$ week & & & $11 \%$ & $19 \%$ & $39.8 \%,<11$ days \\
\hline$<1$ week to 1 month & & & $21 \%$ & $49.4 \%$ & $29.0 \%, 11-30$ days \\
\hline 1 to 6 months & & & $67 \%$ & $31.6 \%$ & $27.6 \%, 31-90$ days \\
\hline \multicolumn{6}{|l|}{ Index event } \\
\hline Stroke & 76.3 & 100 & 66.3 & 78.9 & 100 \\
\hline TIA & 23.7 & & 28.4 & 21.1 & \\
\hline \multicolumn{6}{|l|}{ Stroke subtype } \\
\hline Lacunar & 39.4 & & 50.3 & 52.5 & 52.1 \\
\hline LAA & 27.5 & & 30.5 & 34.0 & 28.6 \\
\hline Cryptogenic & 33.1 & & 19.2 & 9.9 & 15.5 \\
\hline Follow-up time & 2 year & 1.91 years & 3.5 years & 1.5 year & 2.5 years \\
\hline \multicolumn{6}{|l|}{ Vascular risk factors } \\
\hline HTN & 60.5 & 65 & 59.5 & 78.2 & 74 \\
\hline $\mathrm{DM}$ & 15.3 & 25.5 & 18.7 & 68.4 & 28.3 \\
\hline Smoking & 24.1 & 22 & 36.3 & 47.3 & 21.2 \\
\hline Dyslipidemia & 22.9 & 37.5 & 46.4 & 56.3 & 46.6 \\
\hline Previous IS & & 18 & 11.4 & 26.1 & 18.2 \\
\hline MIE Rate (\%/year) & $8.0 \%$ & $7.4 \%$ & $3.6 \%$ & $7.5 \%$ & $5.2 \%$ \\
\hline
\end{tabular}

CAPRIE $=$ Clopidogrel vs Aspirin in Patients at Risk of Ischemic Events trial; DM = Diabetes Mellitus; ESPIRIT = European/Australasian Stroke Prevention in Reversible Ischaemia Trial; ESPS-2 = European Stroke Prevention Study-2; ETR = Event to Randomization; HTN = hypertension; IS = ischemic stroke; LAA= large artery atherosclerosis; MATCH = Management of Atherothrombosis with Colpidogrel in High-Risk Patients trial; MIE= Major Ischemic Event; $\mathrm{N}=$ number of patients; PRoFESS = Prevention Regimen for Effectively Avoiding Second Strokes study; TIA = transient ischemic attack

${ }^{a}$ In ESPS-2 and ESPRIT = unspecified. Major ischemic event rate: nonfatal stroke, nonfatal myocardial infarction, or death

( $p=0.006)$. The result of ESPS2 was much debated as a very low dose of aspirin was used. In addition, there was no reduction in the risk of MI or vascular death. Due to this, the ESPRIT was conducted to resolve the uncertainty.

ESPRIT was an open-label, randomized trial comparing daily aspirin of $30 \mathrm{mg}$ to $325 \mathrm{mg}$ with and without dipyridamole (200 $\mathrm{mg}$ bid) [29]. This trial enrolled 2739 patients who had a history of TIA or minor stroke within the preceding 6 months of enrollment. The primary outcome measure was defined as the composite of death from all vascular causes, nonfatal stroke, nonfatal MI, or major bleeding complications. The median aspirin dose was $75 \mathrm{mg}$ in both treatment groups, and ERDP was used by $83 \%$ of patients on the combination therapy. After a mean follow-up period of 3.5 years, 13 vs $16 \%$ of patients reached the primary outcome favoring the combination of aspirin plus dipyridamole in comparison to aspirin alone (ARR, $1 \%$ per year [95\% CI, 0.1-1.8]; HR, 0.80 [95\% CI, 0.66 to 0.98$]$ ). There was no significant difference in the risk of major hemorrhage between the 2 treatment groups (HR, 0.67 [95\% CI, 0.44-1.03]).
In both the ESPS-2 and the ESPRIT studies, discontinuation of combination therapy was more often than aspirin alone. The most common side effects leading to discontinuation of the combination therapy was headache. Based on these data, combination therapy of aspirin and dipyridamole provides more benefit in secondary stroke prevention when compared to aspirin alone.

\section{Clopidogrel}

The Clopidogrel versus Aspirin in Patients at Risk of Ischemic Events (CAPRIE) trial compared the efficacy of daily doses of clopidogrel $(75 \mathrm{mg}) v s$ aspirin $(325 \mathrm{mg})$ in prevention of ischemic stroke, MI, or vascular death in 19,185 patients with a history of recent MI, symptomatic peripheral arterial disease, or recent ischemic stroke [30]. For a mean follow-up of 1.9 years, $5.32 \%$ per year of patients treated with clopidogrel and $5.83 \%$ per year with aspirin reached the primary outcome (RRR, 8-7\% [95\% CI, 0.3-16.5]; $p=0.043$ ). There was no significant increase in intracranial hemorrhage and there was a 
statistical significant reduction in gastrointestinal hemorrhage in the clopidogrel group. Based on this data, the authors concluded that clopidogrel is more effective than aspirin in reducing the combined risk of ischemic stroke, myocardial infarction, or vascular death in patients with atherosclerotic vascular disease.

\section{Aspirin and Clopidogrel}

Based on the cardiac literature, there is evidence supporting the use of combined aspirin and clopidogrel for prevention of vascular complications in patients with acute MI [31]. The therapeutic benefit of combination therapy in stroke prevention was unclear. Two large randomized controlled trials, the Management of Atherothrombosis with Colpidogrel in High-Risk Patients (MATCH) trial and the Clopidogrel for High Atherothrombosis Risk and Ischemic Stabilization, Management, and Avoidance (CHARISMA) trial were conducted to answer the question $[32,33]$.

The MATCH trial compared the efficacy of the combination of aspirin plus clopidogrel $(75 \mathrm{mg} / 75 \mathrm{mg})$ or clopidogrel $(75 \mathrm{mg})$ monotherapy in prevention of recurrent ischemic vascular events in high-risk patients after TIA or ischemic stroke (Diener et al. [33], 2004), with 7599 patients enrolled. The primary endpoint was defined as a composite of ischemic stroke, myocardial infarction, vascular death, or re-hospitalization for acute ischemia. After an average of 18 months follow-up, 15.7\% of patients receiving aspirin plus clopidogrel reached the primary endpoint compared to $16.7 \%$ in the clopidogrel monotherapy group; this did not reach statistical significance $(p=0.024)$. Both life-threatening hemorrhage and major bleeding were increased in the aspirin plus clopidogrel combination as compared to clopidogrel monotherapy (ARI, 1.26 [95\% CI, 0.64-1.88]; $p<0.0001$ and ARI, 1.36 [95\% CI, 0.86-1.86]; $p<0.0001$, respectively).

The CHARISMA trial examined the effect of combination clopidogrel plus low-dose aspirin $v s$ aspirin monotherapy in prevention of recurrent ischemic vascular events in patients at high risk for atherothrombotic events (Bhatt et al., [32], 2006), with 15,606 patients included who had either clinically evident cardiovascular disease or multiple risk factors. The primary endpoint was defined as a composite of myocardial infarction, stroke, or death from cardiovascular causes. Patients were followed for a median of 28 months with $6.8 \%$ vs $7.3 \%$ patients who reached the primary efficacy endpoint in the clopidogrel plus aspirin $v s$ aspirin monotherapy group $(p=0.22)$. For the endpoint of nonfatal stroke, combination therapy showed significant benefits in comparison to aspirin monotherapy (RR, 0.80; $95 \%$ CI, $0.65-0.997 ; p=0.05)$. Overall the risk of severe bleeding was $1.7 \%$ and $1.3 \%$ in the combination therapy group and the aspirin group, respectively $(p=0.09)$. Based on the MATCH and CHARISMA trials, routine use of the combination of aspirin and clopidogrel for prevention of ischemic vascular events is not recommended.

\section{Aspirin Plus Dypyridimole vs Clopidogrel}

Indirect comparisons suggested that aspirin-ERDP was superior to clopidogrel in the prevention of recurrent stroke [34]. The Prevention Regimen for Effectively Avoiding Second Strokes (PRoFESS) study is a randomized, doubleblind, noninferiority trial with a $2 \times 2$ factorial design comparing aspirin-ERDP $(25 / 200 \mathrm{mg})$ twice daily with clopidogrel $(75 \mathrm{mg})$ daily for prevention of recurrent stroke [35]. A total of 20,332 patients were followed-up for a mean of 2.5 years. Recurrent stroke occurred in $9.0 \%$ of aspirin-ERDP recipients and in $8.8 \%$ clopidogrel recipients (HR, 1.01 [95\% CI, 0.92-1.11]). The composite outcome of stroke, myocardial infarction, or vascular death occurred in $13.1 \%$ in each group (HR, 0.99 [95\% CI, 0.92-1.07]). There were more major hemorrhagic events in the aspirinERDP group than in the clopidogrel group $(4.1 \%$ vs $3.6 \%$, respectively; HR, 1.15 [95\% CI, 1.00-1.32]), including intracranial hemorrhage (1.4\% of aspirin-ERDP $v s 1.0 \%$ of clopidogrel; HR, 1.42 [95\% CI, 1.11-1.83]). The net risk of recurrent stroke or major hemorrhagic event was similar in the two groups $(11.7 \%$ in the aspirin-ERDP $v s 11.4 \%$ in the clopidogrel; HR, 1.03 [95\% CI, 0.95-1.11]). Premature discontinuation of the study drug was significantly more frequent among the aspirin-ERDP group than the clopidogrel group (29.1 vs 22.6\%, respectively; $p<0.001$ ). Based on these data, there is no meaningful difference between aspirinERDP and clopidogrel in secondary stroke prevention.

\section{Antiplatelet Agents in Different Stroke Subtypes}

\section{Large Vessel Atherosclerosis}

Large artery atherosclerosis (LAA) is one of the major etiological subtypes of patients enrolled in recent antiplatelet clinical trials and usually accounts for 20 to $30 \%$ of the study population (see Table 4). However, these groups of patients are different from those that are traditionally represented in major stroke databases, as most clinical trials exclude patients who are candidates for carotid endarterectomy. Large artery atherosclerosis can be subcategorized into extracranial or intracranial atherosclerosis, although this is not differentiated in most clinical trials. In this group of patients, destabilization of atherosclerotic plaques leads to platelet activation and formation of platelet rich clot. These fibrin-platelet rich clots then embolize distally (the so-called "artery-to-artery embolization") resulting in ischemic stroke. In addition, the development of acute thrombosis on superimposed arterial plaques can 
lead to hemodynamic insufficiency and hypoperfusion/ border zone infarct as another stroke mechanism. Medical therapy specifically investigating the effects of antiplatelet agents on recurrent stroke in this group of patients is lacking. Some data has been presented from subgroup analysis from previous clinical trials. We will attempt to summarize these data in the following section.

In ESPS-2, $27.5 \%$ of patients enrolled were classified as having large vessel disease as the qualifying event [36]. In the subgroup analysis of patients with large vessel disease, the HR for a recurrent stroke in patients who received aspirin alone vs placebo was 0.74 (95\% CI, 0.54-1.03), and aspirin-ERDP vs placebo was 0.55 (95\%CI, 0.38-0.80). When aspirin-ERDP was compared to aspirin monotherapy, the HR of recurrent stroke was $0.68(\mathrm{HR}=0.74 ; 95 \% \mathrm{CI}$, 0.51 to 1.08$)$. The data from ESPS-2 suggests that aspirin and combination of aspirin-ERDP reduces risk of recurrent stroke in patients with large vessel disease.

In the subgroup analysis of the PRoFESS study, there was a trend of benefit of clopidogrel in comparison to aspirinERDP, with an event rate of stroke of $9.4 \%$ as compared with $10.5 \%$ in patients who were recruited after a large artery stroke [35]. The comparison in lacunar stroke indicated that the 2 drugs were similar (i.e., clopidogrel [8.2\%] vs combined therapy [7.9\%]). This raises the question of whether clopidogrel is more effective in preventing recurrent vascular events in patients with large vessel atherosclerosis.

For ischemic strokes that are due to symptomatic intracranial atherosclerosis, the Warfarin-Aspirin Symptomatic Intracranial Disease (WASID) study is the only larger clinical trial comparing the effects of aspirin versus warfarin for secondary stroke prevention in patients with symptomatic intracranial atherosclerosis [37]. The WASID study tested warfarin (target INR, 2 to 3) against aspirin (1300 mg daily) in 569 patients with a 50 to $99 \%$ stenosis of a major symptomatic intracranial artery. The patient was followed-up for 2 years. The primary endpoint was defined as ischemic stroke, brain hemorrhage, or death from vascular causes other than stroke. The trial was terminated early due to a significant increased risk of death in the warfarin treated group (4.3\% in aspirin vs $9.7 \%$ in warfarin; HR, 0.46 [95\% CI, 0.23-0.90]; $p=0.02)$. The difference between primary endpoint was not observed (HR, 1.04; 95\% CI, 0.73-1.48). Based on the WASID study, aspirin is the standard medical therapy for patients with symptomatic intracranial stenosis. This trial also highlighted the fact that symptomatic intracranial stenosis have a high recurrent risk of stroke at the rate of approximately $22 \%$ for 18 months.

Whether dual antiplatelet agents may provide more benefit when compared to aspirin alone in intracranial atherosclerosis is unclear. The Clopidogrel plus aspirin for infarction reduction in acute stroke or transient ischemic attack patients with large artery stenosis and microemboli
(CLAIR) study was a randomized, open-label, blindedendpoint trial of the efficacy of clopidogrel (75 $\mathrm{mg}$ daily) plus aspirin (75-60 mg daily) vs aspirin (75-160 mg daily) monotherapy for the reduction of the number of microembolic signals detected with transcranial Doppler ultrasound in patients with a recent stroke (within 7 days of the qualifying event) (Wong, et al. [38], 2010). The trial used microembolic signals as the surrogate marker of stroke risk, with 93 of the 100 patients who had symptomatic intracranial stenosis. The study reported a lower proportion of patients with at least 1 microembolic signal in those in the dual therapy group $v s$ the monotherapy group (RRR, $42.4 \%, 95 \%$ CI, 4.6-65.2; $p=0.025$ ) [38]. Further randomized clinical trials using clinical endpoints, longer followup, and a larger number of patients would be important.

Based on the available data, an antiplatelet agent is recommended in comparison to the use of anticoagulation therapy in large vessel disease (including both extracranial and intracranial atherosclerosis disease). Aspirin, aspirinERDP, and clopidogrel are acceptable options. Whether the combination of aspirin and clopidogrel provides more benefit is unclear. Future trials exploring the efficacy of combination antiplatelet therapy in stroke secondary to large artery atherosclerosis are warranted.

\section{Lacunar Stroke}

Lacunar strokes are small subcortical infarcts that represent more than $25 \%$ of ischemic stroke subtypes in major clinical stroke database and represent approximately $50 \%$ of our recent antiplatelet clinical trial population. Lacunar strokes are a disease that mainly affect small intracranial penetrating arteries [39]. A few distinct pathophysiological processes have been proposed as a cause of this stroke subtypes. One of the more popular mechanisms is lipohyalinosis due to hypertension. Longstanding uncontrolled hypertension causes accelerated arteriolar damage of the small penetrating arteries, which in turn lead to fibrin and collagen deposition in the vessel wall. Whether the final cause of infarct is due to occlusion from fibrin/collagen deposition or formation of superimposed small thrombi is unclear. Another mechanism involves destabilization or plaque rupture of microatheroma formed at the ostium of the small vessel leading to platelet aggregation and thrombus formation as a final pathway of small vessel infarct. Recently, several groups have proposed an intrinsic endothelial dysfunction as an underlying mechanism of lacunar stroke. Based on the previously mentioned proposed pathophysiological processes, antiplatelet agent theoretically is believed to be useful in patients with lacunar stroke subtypes.

There are no clinical trials published that specifically address this stroke subtype. As indicated earlier, due to the inclusion and exclusion criteria, the majority (approximately $50 \%$ ) of the enrolled patients in recent clinical trials have 
small vessel disease as their index events. Some data have been presented from subgroup analysis from these trials. We will attempt to summarize these data in the following section.

The Accidents, IschemiquesCerebraus Lies a l'Atheroscelerose (AICLA) trial is a double-blind randomized controlled trial examining the effect of aspirin or aspirin plus dipyridamole $v s$ placebo on stroke recurrence in patients with a stroke or TIA in the preceding year [40]. Of the 603 patients, 98 with probable small vessel disease were enrolled. In this subgroup of patients, recurrent ischemic stroke occurred in $26 \%$ of placebo, $10 \%$ of aspirin, and $7 \%$ of aspirin plus dipyridamole for 3 years. In ESPS-2, 39.4\% (2600 of 6602) of the patients enrolled were classified to have small vessel disease based on clinical syndromes [36]. The HR for a recurrent stroke in patients with small vessel disease who received aspirin alone $v$ s a placebo was 0.82 (95\% CI, 0.60-1.11), and combination therapy $v s$ a placebo was 0.56 (95\% CI, 0.40 $0.78)$. When combination therapy was compared to aspirin alone, the HR was 0.68 (95\% CI, 0.48-0.97). The data from the ESPS-2 and the AICLA studies suggest that aspirin and a combination of aspirin and dipyridamole reduce the risk of recurrent stroke in patients with small vessel disease.

In the WARSS, 56\% (1237 of 2206) of enrolled patients have been classified as having lacunar stroke $(56.1 \%)$ as the qualifying event [26]. For a 2-year follow-up, patients with lacunar stroke as the index event have a risk of recurrent stroke or death of $15.2 \%$ while on aspirin vs $17.1 \%$ while on warfarin (HR of warfarin $v s$ aspirin, 1.15; 95\% CI, 0.88 to 1.52 ). Although the study was underpowered in this stroke subtype, it did provide some indirect evidence to show that there was no difference in secondary stroke prevention in lacunar stroke between aspirin and warfarin.

In the PRoFESS study, $52 \%(10,578$ of 20,332) of patients enrolled in the trial have lacunar stroke as the index event [35]. In the subgroup analysis involving only patients with lacunar stroke, 418 of $5292(7.9 \%)$ of patients on aspirin-ERDP vs 427 of $5286(8.1 \%)$ of patients on clopidogrel had a recurrent stroke during a 2.5-year follow-up. These data suggest that clopidogrel and aspirin-ERDP provide similar benefits to patient with small vessel disease [35].

Based on the available data, antiplatelet agent is recommended during the use of anticoagulation therapy in lacunar stroke. Aspirin, aspirin-ERDP, and clopidogrel are acceptable options. Whether a combination of aspirin and clopidogrel may provide more benefit is unclear. The Secondary Prevention of Small Subcortical Strokes-3 (SPS-3) trial is a phase III study funded by the National Institutes of Health, which is a double-blind, randomized clinical trial with a $2 \times 2$ factorial design investigating whether: 1) aspirin plus clopidogrel combination therapy is superior to aspirin monotherapy and
2) "intensive" (systolic, $<130 \mathrm{mmHg}$ ) blood pressure control is superior to "usual" (systolic, 130-149 mmHg) in preventing recurrent stroke and major vascular events in 3000 patients with small subcortical stroke. Follow-up will be made for 3 years [41]. This is the first large clinical trial studying lacunar stroke, and hopefully it will provide important insight into the management of lacunar stroke.

\section{Selection of Antiplatelet Agents}

Based on a review of the current literature, the following recommendations can be made regarding the use of antiplatelets in secondary stroke prevention.

For cardioembolic stroke:

1) Anticoagulation remains the treatment of choice.

2) Aspirin is indicated in patients with low risk of stroke (i.e., CHADS score $=0$ or 1 ).

3) Dual antiplatelet agents are indicated in patients who are unsuitable for treatment with oral anticoagulant.

For noncardioembolic stroke:

1) Aspirin is preferred over warfarin.

2) Combined therapy with dipyridamole and aspirin is better than aspirin alone.

3) There is no clinical meaningful difference between clopidogrel and the combined therapy of dipyridamole and aspirin.

4) There is no established role for combined therapy with clopidogrel and aspirin.

5) Selection of aspirin, clopidogrel, or a combination of aspirin and dipyridamole depends on cost of treatment, tolerance of adverse effects, compliance to medications, and other comorbidities.

Because the effect of antiplatelet agents is modest, at best, for secondary stroke prevention, patient education and management of other vascular risk factors (e.g., hypertension, dyslipidemia, and smoking) are equally important.

\section{Antiplatelet Resistance}

The use of aspirin and clopidogrel for secondary stroke prevention has only shown modest clinical efficacy, and stroke recurrences occur even when patients are treated with therapeutic doses of these agents. This phenomenon has been described with a number of terms, including "treatment failure" or "treatment unresponsiveness." Many mechanisms have been proposed, including patient nonadherence, and the presence of comorbid conditions. The concept of "antiplatelet resistance" has also been brought forward as a possible explanation. Antiplatelet resistance occurs when aspirin or clopidogrel biochemically fails to inhibit platelet activation, as measured by in vitro platelet 
function assays [42]. Several different platelet function assays have been studied, and the lack of a standardized assay makes antiplatelet resistance difficult to define. Moreover, even when there is measurable antiplatelet resistance, this correlates poorly with recurrent clinical events. Therefore, presently we do not recommend routine screening of patients for "treatment resistance."

Although there is no standardized measure of antiplatelet resistance, it may be important to suspect resistance in patients with recurrent stroke as it is a potentially modifiable factor. Proposed mechanisms responsible for antiplatelet resistance include drug-drug interactions and genetic polymorphisms of antiplatelet metabolism or drug-receptor site. For instance, nonsteroidal anti-inflammatory drugs (NSAIDS), such as ibuprofen, can reduce the clinical benefit of aspirin in a variety of vascular disease by competing with the COX-2 receptor site to which aspirin binds [43]. Agents that inhibit the CYP3A4 and CYP2C19 enzyme systems can counter the action of clopidogrel by preventing its conversion to active metabolites from a pro-drug state. Atorvastatin has been demonstrated to attenuate the antiplatelet activity of clopidogrel in a dose-dependent manner [44]. However, this has not been shown to affect clinical events in the post hoc analysis of the Clopidogrel for the Reduction of Events During Observation (CREDO) trial [45]. Another possible interaction with clopidogrel involves the proton pump inhibitors, because they are eliminated by CYP2C19. Further studies are still needed to determine if such interactions impact the risk for recurrent stroke.

\section{Important Considerations for the Design of Future}

Antiplatelet Clinical Trials for Secondary Stroke Prevention

As new antiplatelet agents are being developed, many more clinical trials will be conducted to study the efficacy and safety of these agents in secondary stroke prevention. Based on the current review of previous antiplatelet trials, a number of factors are important in the design of future studies.

1) Etiological Subtypes: In most of the recent clinical trials, small vessel disease, large vessel atherosclerosis, and cryptogenic strokes are grouped together and classified as noncardioembolic stroke or stroke of presumed arterial origin. These different etiological subtypes are considered the same in a clinical trial setting; however, in "real life" they behave very differently. Substantial differences have been observed in the 3 major etiological classifications: 1) cardioembolic stroke, 2) lacunar stroke, and 3) large artery atherosclerosis. These differences include their underlying pathophysiology, risk factor profile, vascular comorbidities, natural history, and response to second- ary prevention. Differential effects of medical therapy may exist between different stroke subtypes, but by lumping them together, these effects may be masked. Future studies should be specific for etiological subtypes, as we have seen with the atrial fibrillation and the symptomatic carotid stenosis trials. We should learn from the existing trials, such as the Secondary Prevention of Small Subcortical Strokes-3 (SPS-3) trial and the WASID trial, to better understand the natural history of the subtype of strokes in a clinical trial setting, so that we can improve our current trial design.

2) Coexisting Vascular Risk Factors: Patients' coexisting vascular risk factors have a major impact on the event rate of clinical trials. Those with polyvascular risk factors or with disease of multiple vascular beds (coronary artery disease, peripheral vascular disease, or stroke) have a higher risk of major vascular events. For instance in the MATCH trial, which included highrisk patients with atherothrombotic risk factors, their major ischemic event rate was $7.5 \%$ per year as compared to other trials of the similar period, such as the PRoFESS study and the ESPRIT, which were 5.2\% and $3.6 \%$ per year, respectively (Table 4). The advantage of "enriching" the study population with high-risk vascular patients is that fewer patients may be needed to show a statistical significance, and there is less likelihood of masking treatment benefits due to the low event rate. On the other hand, it has to be assumed that all patients in the study group have similar risk factor management. For the past decade, secondary stroke prevention strategy is no longer limited to antithrombotic agents. Lipid-lowering agents and antihypertensive agents have been shown to provide significant benefit in reducing recurrent vascular events in secondary prevention trials. However, in most clinical trials it is unclear as to how these risk factors are managed and how they may interfere with the interpretation of clinical trial results.

3) Timing from Index Event to Randomization: Recent studies on the natural history of TIAs and minor stroke showed that recurrent risk of stroke is highest within the first week after the initial warning event. Therefore, treatment targeting a specific period (within $24 \mathrm{~h}$ to 1 week) post-TIA may be most beneficial. In the subgroup of patients who were enrolled in the MATCH trial within $<7$ days from the index event, a trend toward benefit of a combination of aspirin plus clopidogrel was observed. Interestingly, there is a trend toward shorter event to randomization time in recent clinical trials, with ESPRIT having only $11 \%$ of patients enrolled within less than 1 week to PRoFESS having $40 \%$ of patients enrolled within the first 10 days of index event. This is an encouraging trend and 
PRoFESS has shown that early randomization is possible in large clinical trials. This should be further explored in a future trial design.

4) Elderly Population: Age is one of the predictors for stroke recurrences and the elderly (age $>75$ years) account for more than half of all strokes. However, until recently there have not been stroke-related prevention trials, such as Birmingham Atrial Fibrillation Treatment of the Aged (BAFTA) and HYpertension in the Very Elderly Trial (HYVET), having studied this specific population. Data on the efficacy of antiplatelet agents for secondary stroke prevention in the elderly population is lacking. The only study that has published a subgroup analysis on this age group is the ESPS-2 study. The EPSP-2 study results suggest that the elderly have a higher stroke recurrence rate, and the efficacy of aspirin, and aspirin plus dipyridamole are similar. Most of the other antiplatelet trials including clopidogrel versus aspirin in patients at risk of ischaemic events (CAPRIE), MATCH, PRoFESS, and ESPIRIT have not provided detailed subgroup analysis on the elderly population. There is a need for clinical trials in this age group and future trial design should take this into consideration.

Acknowledgement Full conflict of interest disclosure is available in the electronic supplementary material for this article.

\section{References}

1. Roger VL, Go AS, Lloyd-Jones DM, et al. Heart disease and stroke statistics - 2011 update: a report from the American Heart Association. Circulation 2011;123:e18-e209.

2. Hill MD, Yiannakoulias N, Jeerakathil T, Tu JV, Svenson LW, Schopflocher DP. The high risk of stroke immediately after transient ischemic attack: a population-based study. Neurology 2004;62:2015-2020.

3. Johnston SC, Gress DR, Browner WS, Sidney S. Short-term prognosis after emergency department diagnosis of TIA. JAMA 2000;284:2901-2906.

4. Adams RJ, Albers G, Alberts MJ, et al. Update to the AHA/ASA recommendations for the prevention of stroke in patients with stroke and transient ischemic attack. Stroke 2008;39:1647-1652.

5. Sacco RL, Adams R, Albers G, et al. Guidelines for prevention of stroke in patients with ischemic stroke or transient ischemic attack: a statement for healthcare professionals from the American Heart Association/American Stroke Association Council on Stroke: co-sponsored by the Council on Cardiovascular Radiology and Intervention: the American Academy of Neurology affirms the value of this guideline. Stroke 2006;37:577-617.

6. Vane JR, Botting RM. The mechanism of action of aspirin. Thromb Res 2003;110:255-258.

7. Humbert M, Nurden P, Bihour C, et al. Ultrastructural studies of platelet aggregates from human subjects receiving clopidogrel and from a patient with an inherited defect of an ADP-dependent pathway of platelet activation. Arterioscler Thromb Vasc Biol 1996;16:1532-1543.

8. Harker LA, Kadatz RA. Mechanism of action of dipyridamole. Thromb Res Suppl 1983;4:39-46.

9. Lovett JK, Coull AJ, Rothwell PM. Early risk of recurrence by subtype of ischemic stroke in population-based incidence studies. Neurology 2004;62:569-573.

10. Petty GW, Brown RD Jr., Whisnant JP, Sicks JD, O'Fallon WM, Wiebers DO. Ischemic stroke subtypes: a population-based study of functional outcome, survival, and recurrence. Stroke 2000;31:1062-1068.

11. Purroy F, Montaner J, Molina CA, Delgado P, Ribo M, AlvarezSabin J. Patterns and predictors of early risk of recurrence after transient ischemic attack with respect to etiologic subtypes. Stroke 2007;38:3225-3229.

12. CAST: randomised placebo-controlled trial of early aspirin use in 20,000 patients with acute ischemic stroke. CAST (Chinese Acute Stroke Trial) Collaborative Group. Lancet 1997;349:16411649.

13. The International Stroke Trial (IST): a randomised trial of aspirin, subcutaneous heparin, both, or neither among 19,435 patients with acute ischemic stroke. International Stroke Trial Collaborative Group. Lancet 1997;349:1569-1581.

14. Chen ZM, Sandercock P, Pan HC, et al. Indications for early aspirin use in acute ischemic stroke: a combined analysis of 40 000 randomized patients from the Chinese acute stroke trial and the international stroke trial. On behalf of the CAST and IST collaborative groups. Stroke 2000;31:1240-1249.

15. Kennedy J, Hill MD, Ryckborst KJ, Eliasziw M, Demchuk AM, Buchan AM. Fast assessment of stroke and transient ischemic attack to prevent early recurrence (FASTER): a randomised controlled pilot trial. Lancet Neurol 2007;6:961-969.

16. Wang Y, Johnston SC. Rationale and design of a randomized, double-blind trial comparing the effects of a 3-month clopidogrelaspirin regimen versus aspirin alone for the treatment of high-risk patients with acute nondisabling cerebrovascular event. Am Heart J 2010;160:380-386.

17. Algra A, van Gijn J. Aspirin at any dose above $30 \mathrm{mg}$ offers only modest protection after cerebral ischaemia. J Neurol Neurosurg Psychiatry 1996;60:197-199.

18. Diener HC, Cunha L, Forbes C, Sivenius J, Smets P, Lowenthal A. European Stroke Prevention Study. 2. Dipyridamole and acetylsalicylic acid in the secondary prevention of stroke. J Neurol Sci 1996;143:1-13.

19. Baigent C, Blackwell L, Collins R, et al. Aspirin in the primary and secondary prevention of vascular disease: collaborative metaanalysis of individual participant data from randomised trials. Lancet 2009;373:1849-1860.

20. Hart RG, Pearce LA, Aguilar MI. Meta-analysis: antithrombotic therapy to prevent stroke in patients who have nonvalvular atrial fibrillation. Ann Intern Med 2007;146:857-867.

21. Connolly S, Pogue J, Hart R, et al. Clopidogrel plus aspirin versus oral anticoagulation for atrial fibrillation in the Atrial Fibrillation Clopidogrel Trial with Irbesartan for Prevention of Vascular Events (ACTIVE W): a randomised controlled trial. Lancet 2006;367:1903-1912.

22. Connolly SJ, Pogue J, Hart RG, et al. Effect of clopidogrel added to aspirin in patients with atrial fibrillation. $\mathrm{N}$ Engl $\mathrm{J}$ Med 2009;360:2066-2078.

23. Beneficial effect of carotid endarterectomy in symptomatic patients with high-grade carotid stenosis. North American Symptomatic Carotid Endarterectomy Trial Collaborators. N Engl J Med 1991;325:445-453.

24. Randomised trial of endarterectomy for recently symptomatic carotid stenosis: final results of the MRC European Carotid Surgery Trial (ECST). Lancet 1998;351:1379-1387. 
25. A randomized trial of anticoagulants versus aspirin after cerebral ischemia of presumed arterial origin. The Stroke Prevention in Reversible Ischemia Trial (SPIRIT) Study Group. Ann Neurol 1997;42:857-865.

26. Mohr JP, Thompson JL, Lazar RM, et al. A comparison of warfarin and aspirin for the prevention of recurrent ischemic stroke. N Engl J Med 2001;345:1444-1451.

27. Halkes PH, van Gijn J, Kappelle LJ, Koudstaal PJ, Algra A. Medium intensity oral anticoagulants versus aspirin after cerebral ischaemia of arterial origin (ESPRIT): a randomised controlled trial. Lancet Neurol 2007;6:115-124.

28. Verro P, Gorelick PB, Nguyen D. Aspirin plus dipyridamole versus aspirin for prevention of vascular events after stroke or TIA: a meta-analysis. Stroke 2008;39:1358-1363.

29. Halkes PH, van Gijn J, Kappelle LJ, Koudstaal PJ, Algra A. Aspirin plus dipyridamole versus aspirin alone after cerebral ischaemia of arterial origin (ESPRIT): randomised controlled trial. Lancet 2006;367:1665-1673.

30. A randomised, blinded, trial of clopidogrel versus aspirin in patients at risk of ischemic events (CAPRIE). CAPRIE Steering Committee. Lancet 1996;348:1329-1339.

31. Mehta SR, Yusuf S, Peters RJ, et al. Effects of pretreatment with clopidogrel and aspirin followed by long-term therapy in patients undergoing percutaneous coronary intervention: the PCI-CURE study. Lancet 2001;358:527-533.

32. Bhatt DL, Fox KA, Hacke W, et al. Clopidogrel and aspirin versus aspirin alone for the prevention of atherothrombotic events. $\mathrm{N}$ Engl J Med 2006;354:1706-1717.

33. Diener HC, Bogousslavsky J, Brass LM, et al. Aspirin and clopidogrel compared with clopidogrel alone after recent ischemic stroke or transient ischemic attack in high-risk patients $(\mathrm{MATCH})$ : randomised, double-blind, placebo-controlled trial. Lancet 2004;364:331-337.

34. Kent DM, Thaler DE. Stroke prevention-insights from incoherence. N Engl J Med 2008;359:1287-1289.
35. Sacco RL, Diener HC, Yusuf S, et al. Aspirin and extended-release dipyridamole versus clopidogrel for recurrent stroke. N Engl J Med 2008;359:1238-1251.

36. Ariesen MJ, Algra A, Kappelle LJ. Antiplatelet drugs in the secondary prevention after stroke: differential efficacy in large versus small vessel disease? A subgroup analysis from ESPS-2. Stroke 2006;37:134-138.

37. Chimowitz MI, Lynn MJ, Howlett-Smith H, et al. Comparison of warfarin and aspirin for symptomatic intracranial arterial stenosis. N Engl J Med 2005;352:1305-1316.

38. Wong KS, Chen C, Fu J, et al. Clopidogrel plus aspirin versus aspirin alone for reducing embolisation in patients with acute symptomatic cerebral or carotid artery stenosis (CLAIR study): a randomised, open-label, blinded-endpoint trial. Lancet Neurol 2010;9:489-497.

39. Benavente O. Antithrombotic therapy in small subcortical strokes (lacunar infarcts). Adv Neurol 2003;92:275-280.

40. Bousser MG, Eschwege E, Haguenau M, et al. "AICLA" controlled trial of aspirin and dipyridamole in the secondary prevention of athero-thrombotic cerebral ischemia. Stroke 1983;14:5-14.

41. Benavente OR, White CL, Pearce L, et al. The Secondary Prevention of Small Subcortical Strokes (SPS3) study. Int J Stroke 2011;6:164-175.

42. Sweeny JM, Gorog DA, Fuster V. Antiplatelet drug "resistance." Part 1: mechanisms and clinical measurements. Nat Rev Cardiol 2009;6:273-282.

43. Catella-Lawson F, Reilly MP, Kapoor SC, et al. Cyclooxygenase inhibitors and the antiplatelet effects of aspirin. NEJM 2001;345:1809 1817.

44. Lau WC, Waskell LA, Watkins PB, et al. Atorvastatin reduces the ability of clopidogrel to inhibit platelet aggregation: a new drugdrug interaction. Circulation 2003;107:32-37.

45. Saw J, Steinhubl SR, Berger PB, et al. Lack of adverse clopidogrel- atorvastatin clinical interaction from secondary analysis of a randomized, placebo-controlled clopidogrel trial. Circulation 2003;108:921-924. 